\title{
Determination of Cefoperazone Sodium in Presence of Related Impurities by Improved Classical Least Squares Chemometric Methods: A Comparative Study
}

\author{
Ibrahim A. Naguib ${ }^{1,2}$ and Hany W. Darwish ${ }^{3,4}$ \\ ${ }^{1}$ Pharmaceutical Chemistry Department, Faculty of Pharmacy, University of Tabuk, Tabuk 71491, Saudi Arabia \\ ${ }^{2}$ Pharmaceutical Analytical Chemistry Department, Faculty of Pharmacy, Beni-Suef University, \\ Alshaheed Shehata Ahmad Hegazy Street, Beni-Suef 62514, Egypt \\ ${ }^{3}$ Department of Pharmaceutical Chemistry, College of Pharmacy, King Saud University, P.O. Box 2457, Riyadh 11451, Saudi Arabia \\ ${ }^{4}$ Analytical Chemistry Department, Faculty of Pharmacy, Cairo University, Kasr El-Aini Street, Cairo 11562, Egypt
}

Correspondence should be addressed to Hany W. Darwish; hdarwish75@yahoo.com

Received 7 January 2016; Revised 29 March 2016; Accepted 21 April 2016

Academic Editor: Pranav S. Shrivastav

Copyright (C) 2016 I. A. Naguib and H. W. Darwish. This is an open access article distributed under the Creative Commons Attribution License, which permits unrestricted use, distribution, and reproduction in any medium, provided the original work is properly cited.

\begin{abstract}
A comparative study is established among 4 chemometric models depending on classical least squares (CLS) approach, namely, spectral residual augmented CLS (SRACLS), net analyte processing CLS (NAP-CLS), orthogonal signal correction CLS (OSC-CLS), and direct orthogonal signal correction CLS (DOSC-CLS). The comparison is expressed through analysis of a case study dataset of UV spectral data of Cefoperazone Sodium (CEF) and its two related impurities: in pure powder form and in pharmaceutical dosage form. Four-level three-factor experimental design was established for optimum analysis. The adopted experimental design gave rise to a training set consisting of 16 mixtures (containing different ratios of interfering species). To test the prediction power of the suggested models, an independent test set consisting of 9 mixtures was used. The presented results show the ability of the proposed models to quantify CEF in presence of two related impurities with high accuracy and selectivity $(103.76 \pm 1.03,102.07 \pm 0.91$, $101.61 \pm 0.72$, and $101.60 \pm 0.72$ for SRACLS, NAP-CLS, OSC-CLS, and DOSC-CLS, resp.). Dosage form analysis results were compared statistically to a published HPLC methodology showing insignificant difference in terms of precision and accuracy, indicating the suggested models reliability and their suitability for quality control analysis of drug product. Compared to other models, OSC-CLS and DOSC-CLS models gave more accurate results with lower prediction error for test set samples.
\end{abstract}

\section{Introduction}

Cefoperazone Sodium (CEF) (Figure 1(a)) [1] belongs to the third-generation cephalosporin group that works via preventing the biosynthesis of bacterial cell wall [2]. According to British Pharmacopeia [3], 7-amino-cephalosporanic acid (7-ACA), (6R,7R)-3-[(acetyloxy)methyl]-7-amino8-oxo-5-thia-1-azabicyclo[4.2.0]oct-2-ene-2-carboxylic acid (Figure 1(b)), and 5-mercapto-1-methyl-tetrazole (5-MER) and 1-methyl-1H-tetrazole-5-thiol (Figure 1(c)) are deemed to be specific impurities for CEF. Pharmacological significance of both impurities was mentioned in literature [4].
A literature review showed a group of analytical methods for CEF quantification in its drug products including spectrophotometry [5, 6], NIR [7], and derivative UV-spectrophotometry for determination of CEF in binary mixtures with sulbactam [8]. Chromatographic methods were utilized for assay of CEF [9], CEF and sulbactam [10, 11]; besides HPLC methodology using $\beta$-cyclodextrin stationary phase for assay of ternary mixture of CEF, ampicillin, and sulbactam was described [12]. Furthermore, LC-MS/MS method was described for assay of CEF and sulbactam binary mixture in plasma [13]. Moreover, electrochemical and voltammetric assay of CEF were stated $[14,15]$. 
<smiles>CCN1CCN(C(=O)N[C@@H](C(=O)N[C@H]2C(=O)N3C(C(=O)O[Na])=C(CSc4nnnn4C)CSC[C@@H]23)c2ccc(O)cc2)C(=O)C1=O</smiles>

(a)<smiles>CC(=O)OCC1=C(C(=O)O)N2C(=O)C[C@H]2SC1</smiles>

(b)<smiles>Cn1nnnc1S</smiles>

(c)

FIgURE 1: Chemical structures of CEF (a) and its reported impurities 7-ACA (b) and 5-MER (c).

CEF and its two mentioned impurities were analyzed by HPLC and HPTLC chromatographic methods [16] and chemometric methods [17].

There are two main aims for the presented work. First, we aim to establish a comparison among 4 chemometric models depending on classical least squares (CLS) approach, namely, spectral residual augmented CLS (SRACLS), net analyte processing CLS (NAP-CLS), orthogonal signal correction CLS (OSC-CLS), and direct orthogonal signal correction CLS (DOSC-CLS). The last 3 models are preprocessing techniques implemented to increase the predictive capabilities of CLS model. The proposed improvement could offer CLS model wider applications for quantitative analysis, yet keeping the advantage of its built-in qualitative properties. The comparison shows the underlying algorithm for each model and compares the analysis results of different mixtures of CEF, 7ACA, and 5-MER, as a case study, to indicate which of the 4 models is best to improve CLS prediction ability. Second, the presented models show the ability of chemometrics to analyze selectively CEF in ternary mixtures with its two reported impurities utilizing inexpensive and available instruments like UV-spectrophotometer, with suggested routine application of the models for quality control analysis of the pharmaceutical dosage form. The selected models offer comparable accuracy and precision for the quantitation of CEF in pharmaceutical formulation compared to the official HPLC method [9].

\section{Materials and Methods}

Full description of instrument, materials, chemical reagents, pharmaceutical formulations, stock, and working preparations applied in the presented study is mentioned in our previous published work [17].

2.1. Linearity. UV spectra for different samples of CEF ranging from 1 to $70 \mu \mathrm{g} \mathrm{mL}^{-1}$ were recorded from 210 to $300 \mathrm{~nm}$. CEF showed linearity between 5 and $50 \mu \mathrm{g} \mathrm{mL}^{-1}$ at its $\lambda_{\max }$ at $229 \mathrm{~nm}$. The superimposed spectra of $10 \mu \mathrm{g} \mathrm{mL}^{-1}$ of CEF, 7-ACA, and 5-MER are shown in Figure 2.

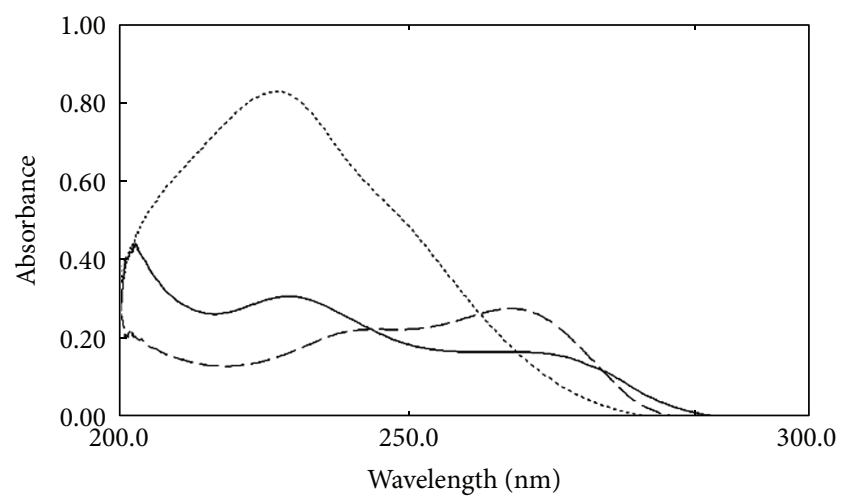

FIGURE 2: Zero-order absorption spectra of $10 \mu \mathrm{g} \mathrm{mL} \mathrm{m}^{-1}$ of CEF (-), 7-ACA (- - ), and 5-MER (......) using methanol as blank.

\subsection{Experimental Design}

2.2.1. Calibration Set. Four-level, three-factor experimental design was accomplished utilizing four concentration levels coded as $+2,+1,-1$, and -2 , where -1 is the central level for CEF and its impurities (7-ACA and 5-MER). The aim of the current design is to cover the mixture space in a proper way, ending up with a training set composed of 16 mixtures [18]. Twenty $\mu \mathrm{g} \mathrm{mL}^{-1}$ of CEF was chosen as a central level for the design and the proposed concentrations for each level for CEF were dependent on its calibration range. Concerning impurities, their concentration levels were based on the fact that we include them in up to $3 \%$ of CEF calculated on molar basis. The concentration design matrix was represented in Table 1. The two-dimensional (2D) scores plot for the first two PCs of the concentration matrix was constructed to check the orthogonality, symmetry, and rotatability for the mixtures of the training set (presented as circles) as shown in Figure 3. The best preprocessing procedure that gives the optimum results was mean centering one.

2.2.2. Test Set. To assess the validity and the predictability of the compared chemometric models, the test set mixtures 
TABLE 1: Four-level three-factor experimental design of the training set (16 mixtures) and test set (9 mixtures) shown as concentrations of the mixture components in $\mu \mathrm{g} \mathrm{mL}^{-1}$.

\begin{tabular}{lccccc}
\hline & Training set & & \multicolumn{3}{c}{ Test set } \\
CEF & 7-ACA & 5-MER & CEF & 7-ACA & 5-MER \\
\hline 18 & 0.13 & 0.065 & 25 & 0.17 & 0.09 \\
18 & 0.15 & 0.07 & 19 & 0.14 & 0.07 \\
20 & 0.15 & 0.09 & 22 & 0.18 & 0.075 \\
20 & 0.2 & 0.07 & 21 & 0.14 & 0.08 \\
26 & 0.15 & 0.065 & 23 & 0.15 & 0.065 \\
20 & 0.13 & 0.085 & 25 & 0.2 & 0.085 \\
18 & 0.18 & 0.085 & 19 & 0.13 & 0.08 \\
24 & 0.18 & 0.07 & 22 & 0.16 & 0.07 \\
24 & 0.15 & 0.085 & 21 & 0.16 & 0.08 \\
20 & 0.18 & 0.065 & & & \\
24 & 0.13 & 0.09 & & & \\
18 & 0.2 & 0.09 & & & \\
26 & 0.2 & 0.085 & & & \\
26 & 0.18 & 0.09 & & & \\
24 & 0.2 & 0.065 & & & \\
26 & 0.13 & 0.07 & & & \\
\hline
\end{tabular}

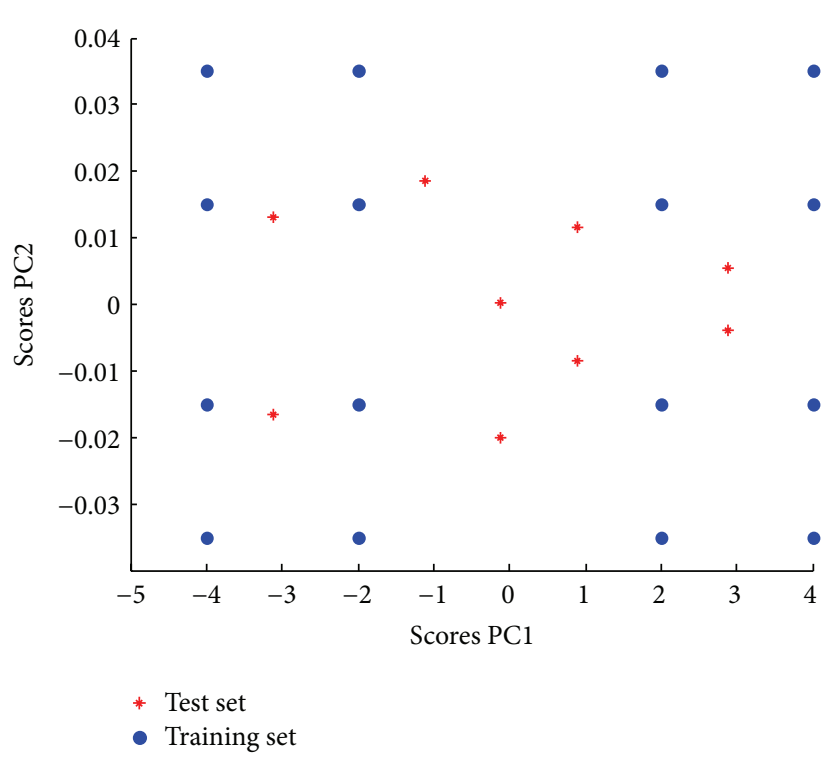

Figure 3: Two-dimensional scores plot for the mean centered 16 training set samples (circles) and the 9 test set samples (stars) of concentration matrices of the 4-level 3-factor experimental design, with \% variance of $99.7 \%$ for first PC and $0.28 \%$ for second PC.

were attained by preparing nine independent mixtures other than the training set mixtures but within the concentration space of the adopted design as indicated in Table 1. The well positioning of the mixtures of both training set (circles) and test set mixtures (stars) is indicated in Figure 3.

2.2.3. Analysis of Cefobid Vial. Working solution of CEF $\left(100 \mu \mathrm{g} \mathrm{mL}^{-1}\right)$ was prepared using methanol as solvent. Lastly, two $\mathrm{mL}$ of this working solution was completed to ten $\mathrm{mL}$ with methanol. The mean of three spectra was measured. This experiment was repeated six times and the attained spectra were analyzed by the suggested models.

2.3. Software. Codes for SRCALS were written with Matlab $^{\circledR}$ 7.1.0.246 (R14). NAP-CLS, OSC-CLS, and DOSC-CLS methods were executed in Matlab 7.1.0.246 (R14) utilizing MVC1 toolboxes [19]. Statistical tests ( $t$-test and $F$-test) were accomplished using Microsoft ${ }^{\circledR}$ Excel.

\section{Chemometric Methods}

3.1. Spectral Residual Augmented Classical Least Squares (SRACLS). Full mathematical description of SRACLS model can be found in literature [20-22].

The new predicted concentration will be calculated according to the following equation:

$$
\widehat{\widetilde{\mathbf{C}}}_{\text {new }}=\mathbf{X} \widehat{\widetilde{\mathbf{K}}}_{\text {new }}^{\prime}\left(\widehat{\widetilde{\mathbf{K}}}_{\text {new }}{\widehat{\widetilde{\mathbf{K}}}_{\text {new }}^{\prime}}^{\prime} \approx \mathbf{X}{\widehat{\widetilde{\mathbf{K}}}_{\text {new }}^{+}}^{+},\right.
$$

where $\widehat{\widetilde{C}}_{\text {new }}$ is the new predicted concentration after necessary augmentation of $\widehat{\mathbf{K}}$ and $\widehat{\widetilde{\mathbf{K}}}_{\text {new }}$ is the augmented pure component contribution which takes into account residual errors, contribution of impurities, and contribution of interfering pure component spectra. The optimum number of loadings used for augmentation of $\widehat{\mathbf{K}}$ matrix was selected through running leave-one-out cross-validation (LOO-CV) [22].

3.2. Improved Signal-CLS Models. CLS, as a direct calibration model, is the simplest developed chemometric technique. CLS method necessitates that all the components in the training set should be well recognized. Contrasting CLS, PCR (principal component regression) and PLS (partial least squares) methods can be applied for determination of the components under inspection even in the presence of unknown interfering component that offered the two models an advantage over CLS [23]. Predictive power of CLS model can be improved greatly by using preprocessing techniques such as the methods proposed in this paper, like net analyte preprocessing (NAP), orthogonal signal correction (OSC), and direct orthogonal signal correction (DOSC). Preprocessing of the data prior to calibration step may be applied to minimize systematic variations influence that is unrelated to the interesting parameters [24].

3.2.1. Net Analyte Preprocessing (NAP). Net analyte signal (NAS) calculation is the basis of NAP. NAS gave rise to numerous new calibration models, based on the same concept, extracting portion of the signal, which is directly correlated to the concentration of analyte and thus beneficial for prediction purposes [25]. Additionally, NAS was applied for calculation of analytical figures of merit and for developing sensor selection technique [25]. The fundamental principle of NAS-based calibration models is to distinguish between two kinds of contributions in the training data matrix $\mathbf{X}$; one originates from the analyte of interest while the second originates from other sources of variability. The full details of NAP are mentioned by Goicoechea and Olivieri [25]. 
3.2.2. Orthogonal Signal Correction (OSC) and Direct Orthogonal Signal Correction (DOSC). Numerous algorithms for applying OSC, as a filtering mechanism to data matrix $\mathbf{X}$, were described in detail in literature [24, 26, 27]. In the presented work, we refer to the OSC algorithm discussed by Fearn [27], as it is the most easily interpreted and can be compared to NAP methodology.

These algorithms are employed to get rid of parts of the spectra that are orthogonal to the concentration. Fearn's algorithm spans the same space as PLS or PCR models on the unprocessed data and extracts components that are strictly orthogonal to the concentration. The first step is a projection of $X$ onto the subspace that is orthogonal to $X^{T} Y_{k}$ to obtain the variation in $X$ that is correlated to $Y_{k}$ (where $Y_{k}$ is concentration of $k$ analyte). Fearn's algorithm removed almost the drawbacks that were seen in the other OSC algorithms such as lack of orthogonality, leading to removal of useful information, or adding useless data in the corrected matrix.

DOSC approach introduced by Westerhuis et al. [28] is based only on least squares steps. It finds components, which are orthogonal to $Y$, which label the main variation of $X$ to be removed from corrected $X$. For implementation of DOSC, firstly $Y$ is decomposed into two orthogonal parts: $\widehat{Y}$ (the projection of $Y$ onto $X$ ) and $F$ (the residual part, i.e., orthogonal to $X)$. Secondly, $X$ is decomposed into two orthogonal parts, one part that is of the same range as $\hat{Y}$ and the other part that is orthogonal to it. Finally principal component analysis (PCA) is applied on the part of $X$ orthogonal to $Y$ to remove it from $X$ giving rise to corrected $X$.

Mathematically, DOSC can be described by the following steps.

Step 1. Step 1 is as follows:

$$
Y=P_{X} Y+A_{X} Y=\widehat{Y}+F
$$

Step 2. Step 2 is as follows:

$$
X=P_{\widehat{Y}} X+A_{\widehat{Y}} X
$$

Step 3. Step 3 is as follows:

$$
X_{\mathrm{DOSC}}=X_{\text {corrected }}=X-t P^{T} \text {, }
$$

where $t$ is score vector and $P$ is loading vector.

The three models (NAP-CLS, OSC-CLS, and DOSC-CLS) are simpler when compared with other methods such as PLS. This simplicity originates from the utilization of orthogonal projections concepts, followed by the well-established classical least squares fitting.

(1) Optimization of Number of Factors for the NAP-CLS, OSCCLS, and DOSC-CLS Models. Leave-one-out cross-validation (LOO-CV) is adopted in the current work for optimization of factors number for construction of the proposed models [23], by building the model using $I-1$ samples' set (15 mixtures from training or calibration set) to predict the one sample left (validation sample). The root mean square error of crossvalidation (RMSECV) is computed as follows:

$$
\mathrm{RMSECV}=\sqrt{\frac{1}{I} \sum_{i=1}^{I}\left(c_{i}-\widehat{c}_{i_{-} \mathrm{CV}}^{A}\right)^{2}},
$$

where $I$ is the number of objects in the calibration set, $c_{i}$ is the known concentration for sample $i$, and $\widehat{c}_{i_{-} \mathrm{CV}}^{A}$ is the predicted concentration of sample $i$ using $A$ components. Mean centering was applied to the calibration set each time successive samples were left out.

\section{Results and Discussion}

The current work was designed to accomplish a number of goals. First goal is to develop simple, selective, and precise chemometric models for assaying CEF in presence of its impurities in pure form and dosage form. Second goal is to demonstrate the quantitative power as well as qualitative power of the suggested models and compare their inherent characteristics using the analyzed mixtures as a case study. Additionally, we aim to display the influence of various preprocessing steps, such as NAP, OSC, and DOSC, on the performance of CLS in quantitative analysis. Optimization of methods' parameters was the first step to run models properly. For the SRACLS model, the optimum number of loadings $P_{\text {new }}$ used for augmentation of $\widehat{\mathbf{K}}$ matrix was selected through running LOO-CV and found to be 4 .

For appropriate building of NAP-CLS, OSC-CLS, and DOSC-CLS methods, number of projection matrix factors (for NAP-CLS) and number of extracted factors (for OSCCLS and DOSC-CLS) were adjusted. For this purpose, LOO$\mathrm{CV}$ was applied where log PRESS (predicted residual error sum of squares) values were computed. The optimum number of factors was chosen in accordance with Haaland and Thomas approach [29]. In all improved CLS models, two factors were essential for constructing the models except in DOSC-CLS model, where three factors were required. This information demonstrates that DOSC, as a preprocessing technique, is more complex than NAP and OSC procedures.

After parameters' optimization and training procedure, all proposed methods, together with ordinary CLS, were applied successfully for estimation of CEF in training set and test set as indicated in Tables 2 and 3, respectively. Recovery percentages, mean recoveries, standard deviation, and RMSEC and RMSEP values are anticipated in Tables 2 and 3.

RMSEC (for training set) and RMSEP (for test set) were computed in a similar way according to the following equation:

$$
\operatorname{RMSEC}(P)=\sqrt{\frac{1}{I} \sum_{i=1}^{I}\left(c_{i}-\widehat{c}_{i}^{A}\right)^{2}}
$$

where $I$ is the number of samples in the test set (in case of RMSEP) and $I-1$ is for training set (in case of RMSEC), $c_{i}$ is the known concentration for sample $i$, and $\widehat{c}_{i}^{A}$ is the estimated concentration of sample $i$ using $A$ components. 


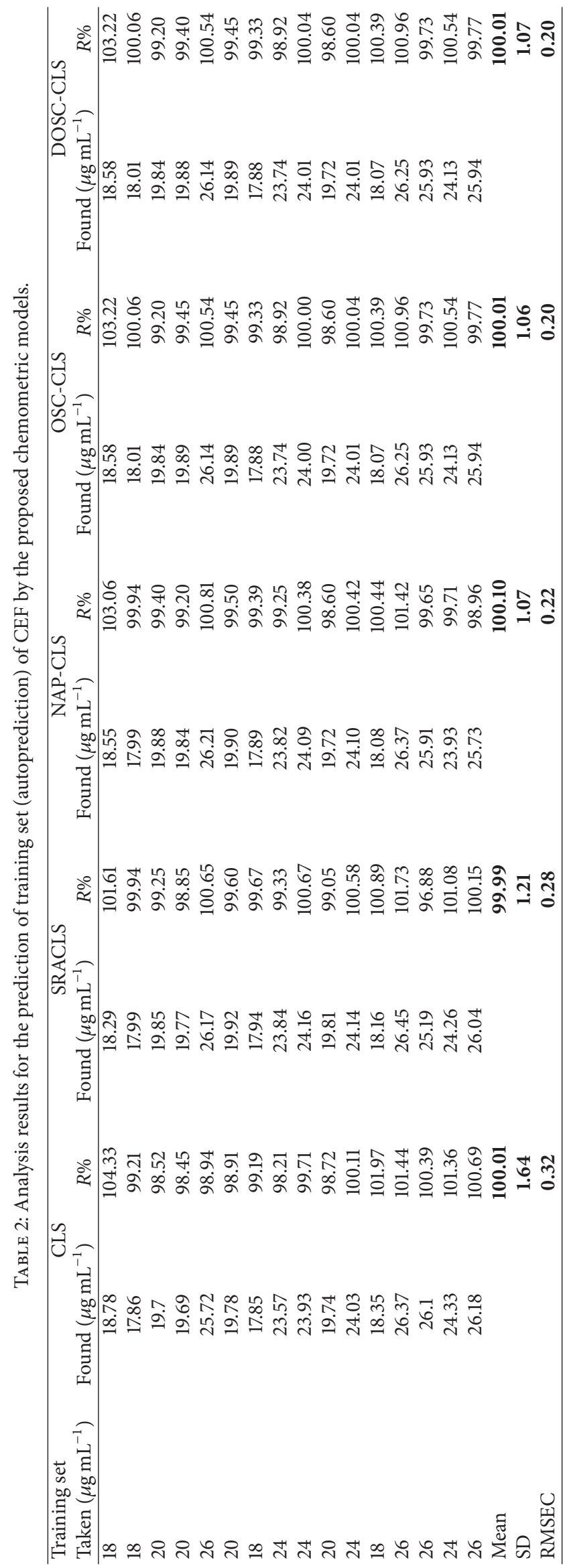




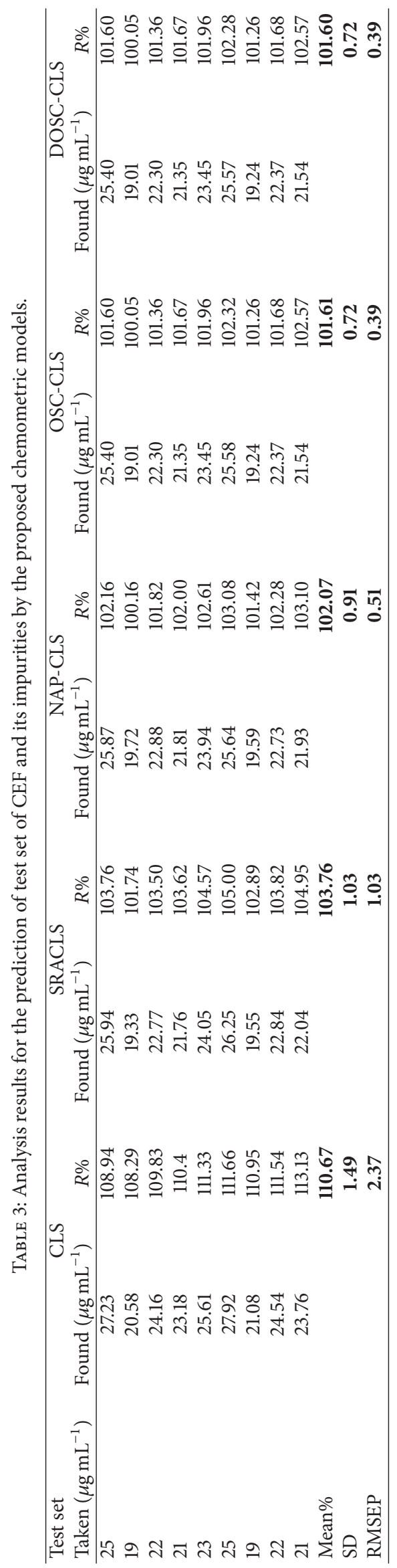


TABLE 4: Statistical comparison of the results obtained by the proposed methods and the reported HPLC method for determination of CEF in pharmaceutical formulation (Cefobid ${ }^{\circledR}$ Vial).

\begin{tabular}{|c|c|c|c|c|c|}
\hline Parameter & SRACLS & NAP-CLS & OSC-CLS & DOSC-CLS & Reported HPLC ${ }^{* *}$ \\
\hline Mean & 102.96 & 104.18 & 104.93 & 104.97 & 102.85 \\
\hline $\mathrm{SD}$ & 1.74 & 0.74 & 1.96 & 1.96 & 1.42 \\
\hline Variance & 3.01 & 0.55 & 3.83 & 3.82 & 2.03 \\
\hline$n$ & 6 & 6 & 6 & 6 & 6 \\
\hline Student's $t$-test (2.228) & 0.119 & 2.017 & 2.097 & 2.145 & - \\
\hline$F$-test $(5.050)$ & 1.485 & 3.673 & 1.889 & 1.889 & - \\
\hline
\end{tabular}

${ }^{* *}$ Reference official method is HPLC [9].

TABLE 5: One-way ANOVA parameters for the different proposed models used for the determination of CEF in Cefobid Vial.

\begin{tabular}{lccccc}
\hline Analyte & Source of variation & DF & Sum of squares & Mean square & $F$-value \\
\hline \multirow{2}{*}{ CEF } & Between exp. & 3 & 15.829 & 5.276 & 2.803 \\
& Within exp. & 20 & 56.069 & $\mathbf{1 . 8 8 2}$ \\
\hline
\end{tabular}

There was no significant difference among the models using one-way ANOVA ( $F$-test), where $F$ tabulated is 3.098 at $p<0.05$.

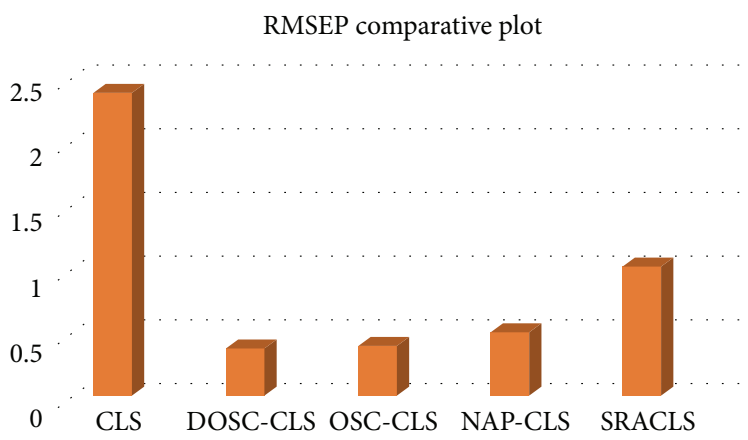

FIGURE 4: RMSEP comparative plot for the prediction of test set samples: 1-SRACLS, 2-NAP-CLS, 3-OSC-CLS, 4-DOSC-CLS, and 5CLS models.

The small values of the RMSEP indicate the minor error of prediction and the high predictive ability of the developed models. Figure 4 shows the RMSEP comparative plot for the prediction of test set samples by the proposed models, where all of the four proposed models are performing better than CLS and best results are given by OSC-CLS and DOSC-CLS models.

The described models were then applied with a great success for the analysis of the available dosage form (Table 4). This fact was further confirmed by the statistical comparison of the suggested models to the official HPLC method [9] (Table 4 ) where the calculated $t$ and $F$ values are less than the tabulated ones, indicating that there is no significant difference between our models and the reference method regarding both accuracy and precision. All the models were additionally compared by one-way ANOVA (Table 5), where the calculated $F$-value was less than the tabulated one as well, showing that there is no significant difference among all models regarding precision. The obtained results suggest the validity of proposed models to be used for routine quality control analysis of CEF in pure form and pharmaceutical product.

\section{Conclusion}

In the presented paper, different chemometric models were applied for the analysis of CEF in presence of its related impurities. The proposed models are CLS, SRACLS, NAPCLS, OSC-CLS, and DOSC-CLS. The developed models combine advantages of rapidity and easiness of traditional spectrometric methods in addition to their quantitative power (prediction of concentrations of CEF in its mixtures). The traditional CLS model gave the worst results for prediction of independent test set samples, while, among the proposed models, OSC-CLS and DOSC-CLS were the most powerful ones that increase the quantitative power of CLS method based on the analyzed case study. All the suggested models were optimized and validated by prediction of test set samples. The methods can be applied for routine quality control analysis of Cefobid Vials without prior separation or interference from commonly encountered additives.

\section{Competing Interests}

No conflict of interests exists in the present paper.

\section{Acknowledgments}

The authors would like to extend their sincere appreciation to the Deanship of Scientific Research at King Saud University for its funding of this research through the Research Group Project no. RGP-322. Additionally the authors would like to extend their sincere appreciation to Dr. Essraa A. Hussein for helping in preparation of the chemical data.

\section{References}

[1] M. J. O'Neil, The Merck Index, An Encyclopedia of Chemicals, Drugs and Biologicals, Merck and Co, Whitehouse Station, NJ, USA, 14th edition, 2013.

[2] J. E. F. Reynolds, Martindale, The Extra Pharmacopoeia, Royal Pharmaceutica Society, London, UK, 31st edition, 1996. 
[3] British Pharmacopeia, Her Majesty's Stationary Office, London, UK, 1980

[4] Q. Tan, Q. Song, and D. Wei, "Single-pot conversion of cephalosporin C to 7-aminocephalosporanic acid using cell-bound and support-bound enzymes," Enzyme and Microbial Technology, vol. 39, no. 5, pp. 1166-1172, 2006.

[5] M. Senthilraja and P. Sanjaypai, "Spectrophotometric method for the determination of cefoperazone sodium in pharmaceutical formulations," Indian Journal of Pharmaceutical Sciences, vol. 68, no. 3, pp. 384-385, 2006.

[6] L. Chen, O. Sha, W.-J. Zhai, and J.-H. Yang, "Fading spectrophotometric determination of cefoperazone sodium in injections by $\mathrm{Fe}^{3+}$-sulfosalicylic acid system," Asian Journal of Chemistry, vol. 25, no. 14, pp. 7918-7920, 2013.

[7] H.-H. Pang, Y.-C. Feng, C.-Q. Hu, and B.-R. Xiang, "Construction of universal quantitative models for determination of cefoperazone sodium for injection from different manufacturers using near infrared reflectance spectroscopy," Guang Pu Xue Yu Guang Pu Fen Xi, vol. 26, no. 12, pp. 2214-2218, 2006.

[8] A. Parra, J. Garcia-Villanova, V. Rodenas, and M. D. Gomez, "First and second derivative spectrophotometric determination of cefoperazone and sulbactam in injections," Journal of Pharmaceutical and Biomedical Analysis, vol. 12, no. 5, pp. 653-657, 1994.

[9] The United States Pharmacopoeia (USP30) National Formulary (NF25), The United States Pharmacopeial Convention, Rockville, Md, USA, 2007.

[10] F. S. Li, Z. X. Xu, H. B. Xiao, and X. M. Liang, "Simultaneous determination of sulbactam sodium and cefoperazone sodium in sulperazon by high performance liquid chromatography,' $S e$ $P u$, vol. 18 , no. 6, pp. 525-526, 2000.

[11] K. V. Kumar, J. Dharuman, and A. K. Sree, "RP-HPLC method development and validation for simultaneous estimation of sulbactam and cefoperazone in dosage form and in plasma," International Journal of Pharma and Bio Sciences, vol. 1, no. 4, article 12, pp. 88-96, 2010.

[12] T.-L. Tsou, Y.-C. Huang, C.-W. Lee, A.-R. Lee, H.-J. Wang, and S.-H. Chen, "Simultaneous determination of ampicillin, cefoperazone, and sulbactam in pharmaceutical formulations by HPLC with $\beta$-cyclodextrin stationary phase," Journal of Separation Science, vol. 30, no. 15, pp. 2407-2413, 2007.

[13] Y. Zhou, J. Zhang, B. Guo et al., "Liquid chromatography/tandem mass spectrometry assay for the simultaneous determination of cefoperazone and sulbactam in plasma and its application to a pharmacokinetic study," Journal of Chromatography B, vol. 878, no. 30, pp. 3119-3124, 2010.

[14] E. Hammam, M. A. El-Attar, and A. M. Beltagi, "Voltammetric studies on the antibiotic drug cefoperazone: quantification and pharmacokinetic studies," Journal of Pharmaceutical and Biomedical Analysis, vol. 42, no. 4, pp. 523-527, 2006.

[15] B. Dogan, A. Golcu, M. Dolaz, and S. A. Ozkan, "Electrochemical behaviour of the bactericidal cefoperazone and its selective voltammetric determination in pharmaceutical dosage forms and human serum," Current Pharmaceutical Analysis, vol. 5, no. 2, pp. 179-189, 2009.

[16] E. A. Abdelaleem, I. A. Naguib, H. E. Zaazaa, and E. A. Hussein, "Development and validation of HPLC and HPTLC methods for determination of cefoperazone and its related impurities," Journal of Chromatographic Science, vol. 54, no. 2, pp. 179-186, 2015.
[17] I. A. Naguib, E. A. Abdelaleem, H. E. Zaazaa, and E. A. Hussein, "Determination of cefoperazone sodium in presence of related impurities by linear support vector regression and partial least squares chemometric models," Journal of Analytical Methods in Chemistry, vol. 2015, Article ID 593892, 8 pages, 2015.

[18] R. G. Brereton, "Multilevel multifactor designs for multivariate calibration," Analyst, vol. 122, no. 12, pp. 1521-1529, 1997.

[19] A. C. Olivieri, H. C. Goicoechea, and F. A. Iñón, "MVC1: an integrated MatLab toolbox for first-order multivariate calibration," Chemometrics and Intelligent Laboratory Systems, vol. 73, no. 2, pp. 189-197, 2004.

[20] D. M. Haaland and D. K. Melgaard, "New prediction-augmented classical least-squares (PACLS) methods: application to unmodeled interferents," Applied Spectroscopy, vol. 54, no. 9, pp. 1303-1312, 2000.

[21] W. Saeys, K. Beullens, J. Lammertyn, H. Ramon, and T. Naes, "Increasing robustness against changes in the interferent structure by incorporating prior information in the augmented classical least-squares framework," Analytical Chemistry, vol. 80, no. 13, pp. 4951-4959, 2008.

[22] I. A. Naguib, "Stability indicating analysis of bisacodyl by partial least squares regression, spectral residual augmented classical least squares and support vector regression chemometric models: a comparative study," Bulletin of Faculty of Pharmacy, Cairo University, vol. 49, no. 2, pp. 91-100, 2011.

[23] R. Kramer, Chemometric Techniques for Quantitative Analysis, CRC Press, 1998.

[24] S. Wold, H. Antti, F. Lindgren, and J. Öhman, "Orthogonal signal correction of near-infrared spectra," Chemometrics and Intelligent Laboratory Systems, vol. 44, no. 1-2, pp. 175-185, 1998.

[25] H. C. Goicoechea and A. C. Olivieri, "A comparison of orthogonal signal correction and net analyte preprocessing methods. Theoretical and experimental study," Chemometrics and Intelligent Laboratory Systems, vol. 56, no. 2, pp. 73-81, 2001.

[26] J. Sjöblom, O. Svensson, M. Josefson, H. Kullberg, and S. Wold, "An evaluation of orthogonal signal correction applied to calibration transfer of near infrared spectra," Chemometrics and Intelligent Laboratory Systems, vol. 44, no. 1-2, pp. 229-244, 1998.

[27] T. Fearn, "On orthogonal signal correction," Chemometrics and Intelligent Laboratory Systems, vol. 50, no. 1, pp. 47-52, 2000.

[28] J. A. Westerhuis, S. de Jong, and A. K. Smilde, "Direct orthogonal signal correction," Chemometrics and Intelligent Laboratory Systems, vol. 56, no. 1, pp. 13-25, 2001.

[29] D. M. Haaland and E. V. Thomas, "Partial least-squares methods for spectral analyses. 1. Relation to other quantitative calibration methods and the extraction of qualitative information," Analytical Chemistry, vol. 60, no. 11, pp. 1193-1202, 1988. 

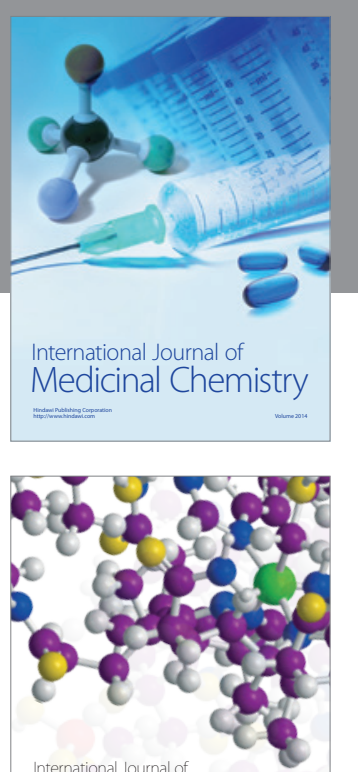

Carbohydrate Chemistry

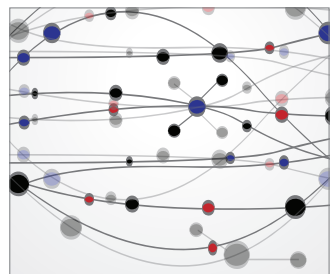

The Scientific World Journal
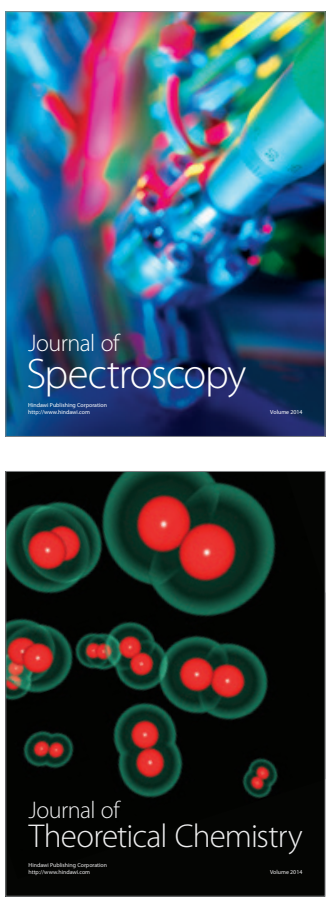
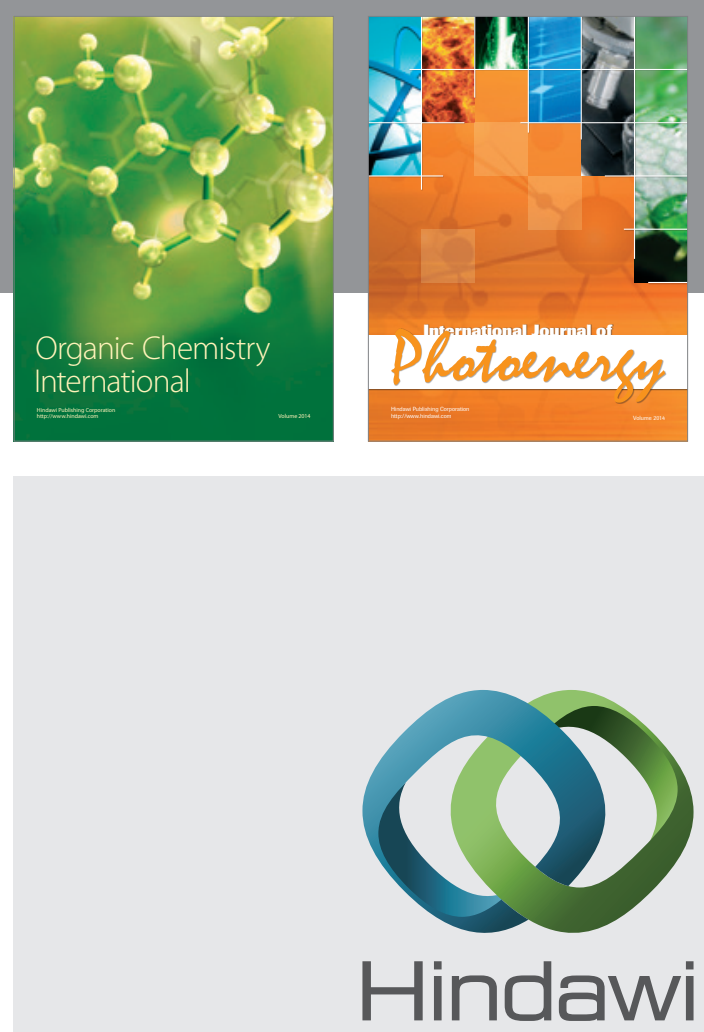

Submit your manuscripts at

http://www.hindawi.com

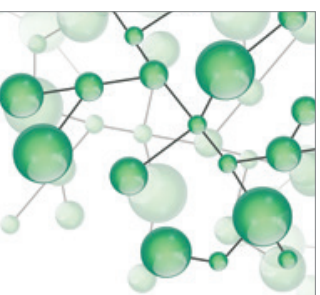

International Journal of

Inorganic Chemistry

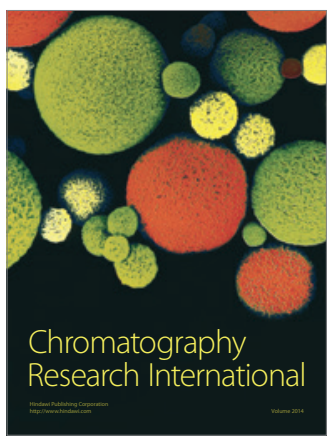

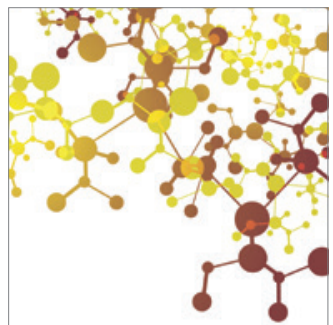

Applied Chemistry
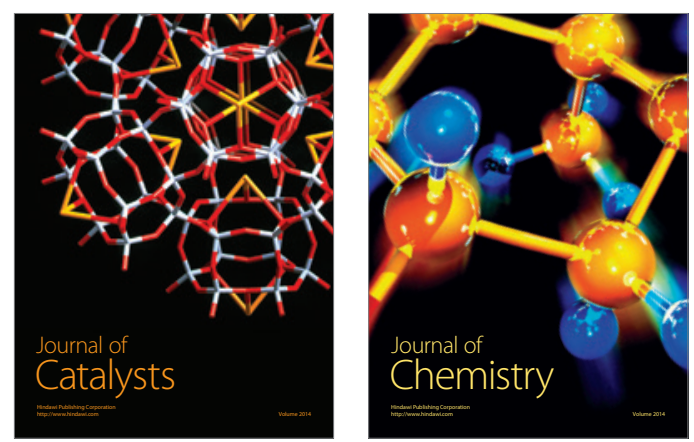
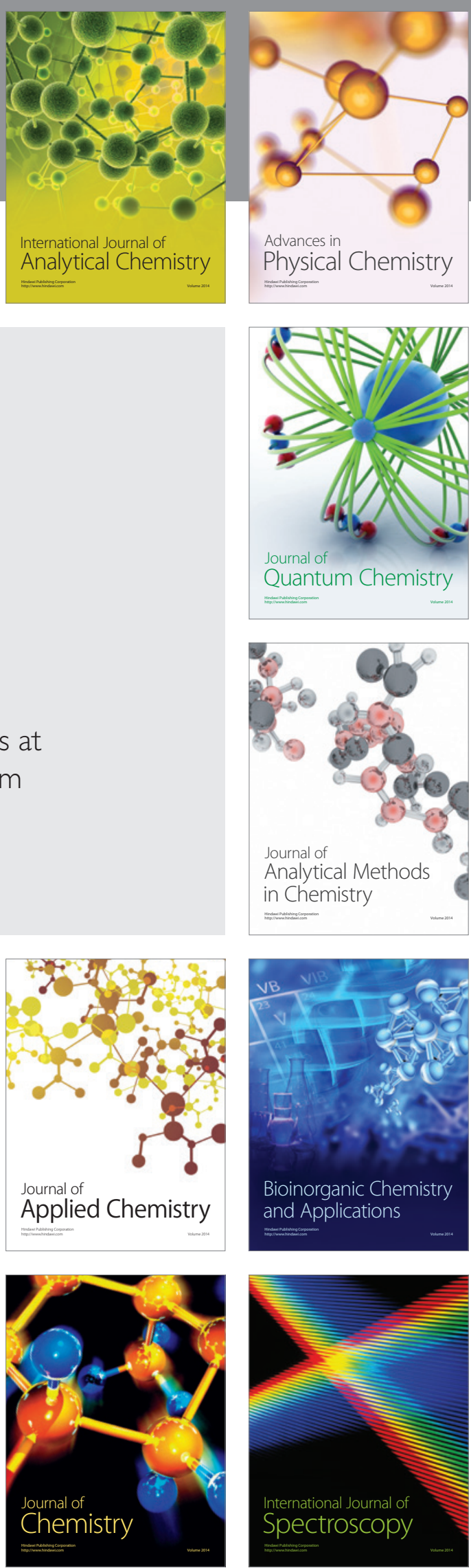